\title{
Invited Discussion on: Correction of High-Grade Pseudogynecomastia After Massive Weight Loss: Modified Inferior Dermoglandular Pedicled Transverse Scar Reduction
}

\author{
Jonathan P. Brower ${ }^{1} \cdot$ J. Peter Rubin ${ }^{1}$
}

Received: 22 October 2019/Accepted: 24 November 2019/Published online: 13 December 2019

(C) Springer Science+Business Media, LLC, part of Springer Nature and International Society of Aesthetic Plastic Surgery 2019

Level of Evidence $V$ This journal requires that authors assign a level of evidence to each article. For a full description of these Evidence-Based Medicine ratings, please refer to the Table of Contents or the online Instructions to Authors www.springer.com/00266.

The authors present a retrospective review of 58 patients who were treated for high-grade pseudogynecomastia resulting from massive weight loss (MWL) with mastectomy and nipple preservation on an inferiorly based dermoglandular pedicle. They assert that the alternativemastectomy with nipple-areola complex (NAC) graftingcarries considerable disadvantages, including loss of nipple sensitivity, hypopigmentation, and a "stuck-on" appearance. These shortcomings, they argue, may be mitigated by their described dermal pedicle technique. The authors are commended for providing a detailed description of their technique which has achieved consistent aesthetic results, high rates of patient satisfaction, and few complications.

Similar to classification systems for gynecomastia [1], a treatment algorithm for pseudogynecomastia has been previously described by our own group in 2008 [2]. A modification to this system, published by Ziegler et al. in 2018 [3], was employed by the authors for the purposes of their study. Patients with grade 1 pseudogynecomastia (minimal tissue excess, preserved NAC position and inframammary fold, IMF) are sufficiently treated with ultrasonic liposuction. A lateral chest skin roll, when present in these circumstances, is generally amenable to direct excision. Grade 2 pseudogynecomastia is characterized by

\section{J. Peter Rubin}

rubipj@upmc.edu

1 Department of Plastic Surgery, University of Pittsburgh Medical Center, Scaife Hall, Suite 6B, 3550 Terrace Street, Pittsburgh, PA 15261, USA a NAC and IMF below their ideal positions at the lower border of the pectoralis major with minimal upper abdominal tissue laxity. This deformity generally benefits from mastectomy with pedicled nipple reconstruction. The most severe cases of pseudogynecomastia are designated as grade 3. Characteristically, the NAC and IMF are below their ideal positions, with concomitant laxity of the upper abdominal skin. These patients are best treated with mastectomy and nipple grafting.

We have used a similar technique for pedicled NAC preservation in our own practice and agree that it can provide an excellent result in select patients, as long as key safety and aesthetic considerations are made (Fig. 1). The authors appropriately excluded patients who had profound weight loss (more than $82 \mathrm{~kg}$ ) because of the inherent difficulty in creating a pedicle of appropriate dimensions. Pedicle design must ensure both adequate perfusion to the NAC while not compromising the aesthetic result with too much bulk buried beneath the skin [4]. Readers are further advised to avoid this technique in patients with active nicotine exposure; the only patient in our published series with delayed healing was covertly smoking cigarettes immediately postoperatively.

Pseudogynecomastia is a central, but not necessarily isolated aesthetic deformity of the upper body in male MWL patients. Accordingly, the authors' description of their surgical technique would benefit from a discussion of methods to incorporate other upper body contouring procedures into the surgical plan. The senior author (JPR) has found that this procedure nicely complements a brachioplasty, as the incisions can be merged within the axilla to contour the lateral chest (see figure). Other details of the technique used by the senior author of this commentary are presented below for comparison. 


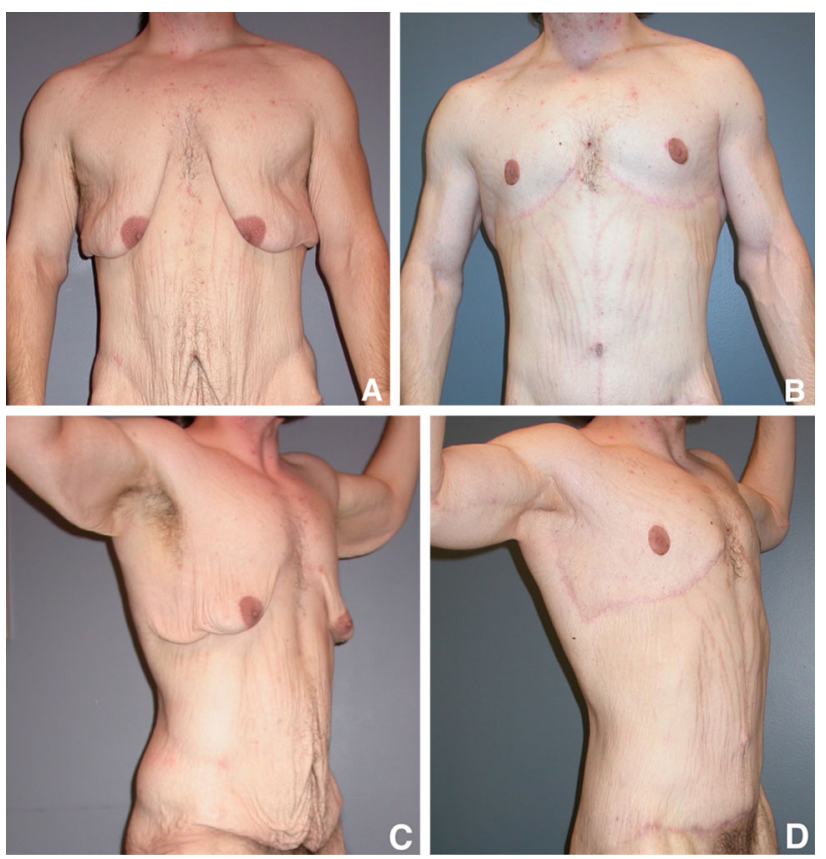

Fig. 1 Pre- and postoperative photographs demonstrating a 30-yearold patient who sustained a $100 \mathrm{lb}$ weight loss and subsequently underwent pseudogynecomastia excision with NAC preservation on a dermal pedicle, as well as brachioplasty. a Pre-op anterior view, b post-op anterior view, c pre-op oblique view, d post-op oblique view (case presented by senior author, JPR)

The authors address optimal scar placement in the IMF crease at the origin of the pectoralis major, but do not include important measures undertaken to achieve an inconspicuous scar. We opt to commit to the superior incision early and anchor it to the deep fascia as described by Lockwood [5]. This technique substantially reduces tension across the dermis and superficial fascial system, thereby minimizing the risk of scar widening and hypertrophy.

Repositioning the nipple from its abnormally medialized position is an important step in addressing pseudogynecomastia in the MWL patient and achieving a natural-appearing result. Numerous methods for determining optimal NAC position are described in the literature as they relate to gynecomastia excision and as well as chest masculinization for gender affirmation [6, 7]. The authors use measurements based on anatomic landmarks to approximate the location for the new nipple, while we prefer to place the NAC at the intersection of the lateral border of the pectoralis and the fifth intercostal space. We have found that leaving $3-4 \mathrm{~cm}$ of skin between the NAC and IMF entirely untouched by scars produces an optimal result. The selected NAC location is marked preoperatively to ensure landmarks are not confounded by positioning on the operating table, and to involve the patient in surgical planning.
To create a NAC that is the appropriate size, we reduce the areola to $2.8 \mathrm{~cm}$ in diameter and deliver it through a circular incision in the skin. The circle is incised at a diameter of $2.0 \mathrm{~cm}$, as this opening will stretch under the tension of the surrounding tissues to approximately $2.5 \mathrm{~cm}$ in diameter. The back of a 10-cc syringe plunger is an ideal template for marking this circular incision [2]. Taken together, these two techniques create a NAC with an anatomic location and shape.

As with any aesthetic procedure, patient satisfaction is of paramount importance when evaluating surgical results. The robust outcomes research that has promulgated the medical literature, including in plastic surgery (BREASTQ [8], BODY-Q [9]), has armed all investigators with the tools necessary to reliably study quality-of-life outcomes and patient satisfaction. By eliciting simple binary patient satisfaction ratings at unclear follow-up intervals, the authors have missed an opportunity to more comprehensively evaluate whether our framework for addressing aesthetic deformities of the male chest is adequate. The previously mentioned validated instruments could be employed to more rigorously analyze patient-reported outcomes to ensure that we are appropriately addressing patients' concerns.

The authors are commended for their effective and safe implementation of an advanced technique for pseudogynecomastia correction with admirable results. We note the paucity of existing literature critically analyzing methods for management of pseudogynecomastia in MWL patients and appreciate the technical description provided. We have been pleased with the results of this technique in our own practice, but do note important limitations. Specifically, the procedure takes considerably more time than it does with nipple grafting and can add significant tissue bulk to the chest. We look forward to future studies that will further enumerate technical considerations and patient satisfaction measures to ensure consistent, aesthetically optimal results.

Funding The authors received no financial support for the research, authorship, and publication of this article.

\section{Compliance with Ethical Standards}

Conflict of interest The authors declared no potential conflicts of interest with respect to the research, authorship, and publication of this article.

Human and animal rights This article does not contain any studies with human participants or animals performed by any of the authors.

Informed consent For this type of study, informed consent is not required. 


\section{References}

1. Waltho D, Hatchell A, Thoma A (2017) Gynecomastia classification for surgical management: a systematic review and novel classification system. Plast Reconstr Surg 139:638e-648e

2. Gusenoff JA, Coon D, Rubin JP (2008) Pseudogynecomastia after massive weight loss: detectability of technique, patient satisfaction, and classification. Plast Reconstr Surg 122:1301-1311

3. Ziegler UE, Lorenz U, Daigeler A, Ziegler SN, Zeplin PH (2018) Modified treatment algorithm for pseudogynecomastia after massive weight loss. Ann Plast Surg 81:290-294

4. Ridha H, Colville RJI, Vesely MJJ (2009) How happy are patients with their gynecomastia reduction surgery? J Plast Reconstr Aesthet Surg 62:1473-1478

5. Lockwood T (1999) Reduction mammaplasty and mastopexy with superficial fascial system suspension. Plast Reconstr Surg 103:1411-1420

6. Atiyeh BS, Dibo SA, El Chafic AH (2009) Vertical and horizontal coordinates of the nipple-areola complex position in males. Ann Plast Surg 63:499-502
7. Mett TR, Krezdom N, Luketina R, Boyce MK, Henseler H, Ipaktchi R, Vogt PM (2017) Optimal positioning of the nippleareola complex in men using the mohrenheim-estimated-tangential-tracking-line (mett-line): an intuitive approach. Aesthetic Plast Surg 41:1295-1302

8. Cohen WA, Mundy LR, Ballard TN, Klassen A, Cano SJ, Browne J, Pusic AL (2016) The BREAST-Q in surgical research: a review of the literature 2009-2015. J Plast Reconstr Aesthet Surg 69:149-162

9. Klassen AF, Cano SJ, Kaur M, Breitkopf T, Pusic AL (2017) Further psychometric validation of the BODY-Q: ability to detect change following bariatric surgery weight gain and loss. Health Qual Life Outcomes 15:227-233

Publisher's Note Springer Nature remains neutral with regard to jurisdictional claims in published maps and institutional affiliations. 